(C) 1984. The Genetical Society of Great Britain

\title{
ELIMINATION OF AN INTRODUCED $B$-CHROMOSOME FROM A WILD POPULATION OF ARCYPTERA FUSCA (Orthoptera)
}

\author{
J. GOSALVEZ AND C. LOPEZ-FERNANDEZ \\ Departmento de Genética, C-XV. Facultad de Ciencias, Universidad Autónoma de Madrid. \\ Madrid-34, Spain
}

Received 13.ii.84

\section{SUMMARY}

Males from a population of the mountain grasshopper Arcyptera fusca possessing a persistent $B$ chromosome were transferred to a population without $B s$ in two successive years. The $B$ was present in the second year, but now appears to have been eliminated. A total of seven successive generations have been analysed.

\section{INTRODUCTION}

Supernumerary chromosomes are widespread in many species of plants and animals. They usually occur only in some populations and in varying frequencies (Jones and Rees, 1982). A majority of the supernumerary systems known in nature are characterised by boosting mechanisms which lead to increases in the frequency of $B \mathrm{~s}$ in the progeny of $B$-containing individuals. It seems that such boosting system can permit $B$ s which have deleterious effects to be maintained for many generations in a population. In fact, if such a boosting system exists, the critical reproductive fitness of a $B$-containing animal permitting the $B$ to be maintained in a population is not 1 but some value less than 1 , depending on the accumulation mechanism. Rates of transmission of $B$ chromosomes have been well studied in some species of grasshoppers by comparing the karyotypes of both parents and progeny from single matings in laboratory conditions (Lucov and Nur, 1973, Hewitt, 1973) and some detailed studies concern the movement of $B s$ in natural populations (Shaw, 1983a, b). However, the ability of a genome to receive and incorporate extra material under natural conditions is difficult to test since unknown factors may condition the survival of this extra material.

The present report concerns the examination during seven consecutive years of two selected natural populations (with and without $B$ s) where individuals containing $B$ s were inoculated into the population without $B \mathrm{~s}$.

\section{Methods}

Meiotic chromosomes were analysed in squash preparations of single follicles fixed in a (1:3) glacial acetic acid: ethanol mixture.

\section{Results AND Discussion}

During the past seven years we have analysed the structure of the chromosome system which exists in different populations of the mountain 


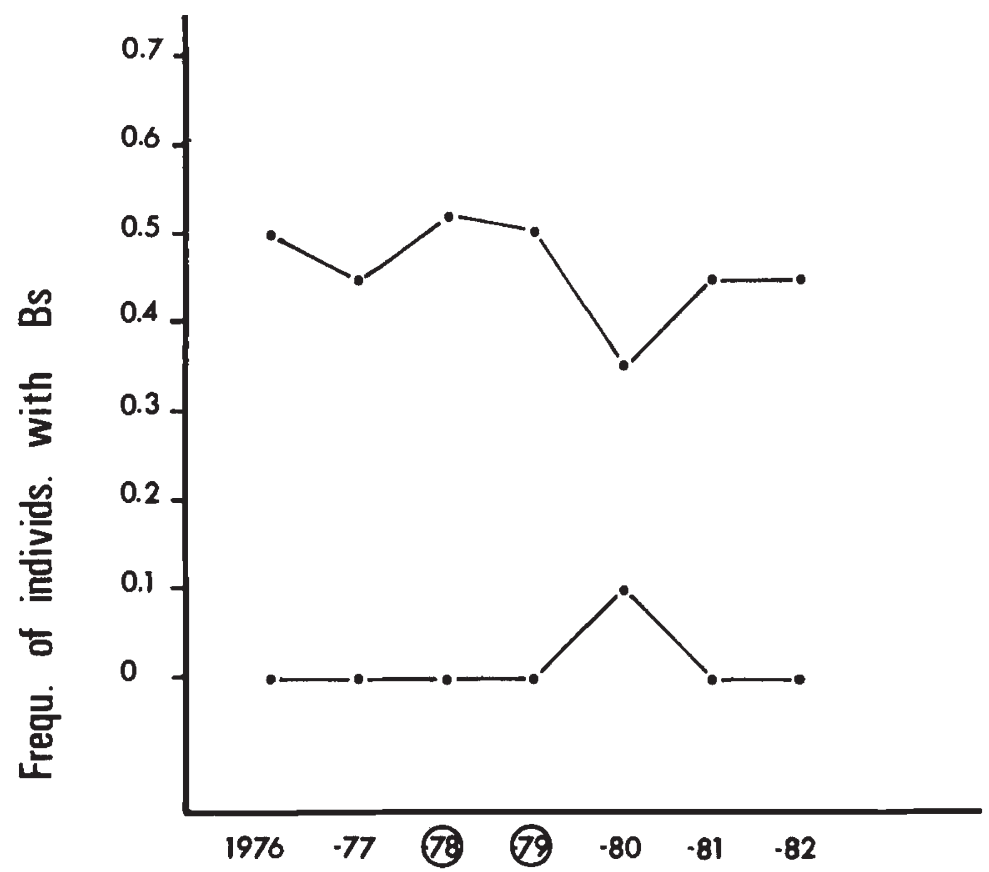

FIG. 1. Variation in the frequency of males containing $B$ s through the different generations. Circles indicate the year of the inoculation. Respumoso's population (upper); Pto de la Bonaigua's population (lower).

grasshopper Arcyptera fusca (Pall.) $(2 \mathrm{n}=22+\mathrm{X} \delta ; 2 \mathrm{n}=22+\mathrm{XX}$ \%) (LópezFernández and Gosálvez, 1981; López-Fernández et al., 1983; LópezFernández and Gosálvez, 1983). Some of these studies have shown the existence of a high frequency of individuals carrying a small mitotically and meiotically unstable $B$ chromosome in a little valley in the Pyrenees (Valle de Respumoso). Populations outside this area are devoid of these supernumeraries. Some populations of Respumoso contain 50 per cent of individuals carrying $B \mathrm{~s}$ in their genomes and this frequency does not seem to vary from generation to generation (table $1 ; \chi_{6}^{2}=2.22 ; \mathrm{P}<0.09$ ). $130 \mathrm{Km}$ eastward from this valley, in Valle de Arán, populations of this species lack $B s$, as do in most of the populations in the Pyrenees. In the summer of 1978 (15th August) one of these populations (Pto. de la Bonaigua) was selected to receive fifty young males from Respumoso; the same operation was carried out during the first days of August in 1979. In this second inoculation young males from Respumoso were captured, marked with a white spot in the pronotum and released in a selected square $(25 \times 25 \mathrm{~m})$ in order to avoid the possibility of recapturing transplanted males. This second inoculation was planned to be done when the population was so young that most of the females were subadult. Thus, we were increasing the possibility of mating between virgin females and inoculated males. This was also favoured because two weeks later unmarked adults males (originated from 1979 generation in Pto. de la Bonaigua) were captured from the same selected square. In order to test the presence of $B$ s transmitted in the previous generation, these individuals were karyotyped. 

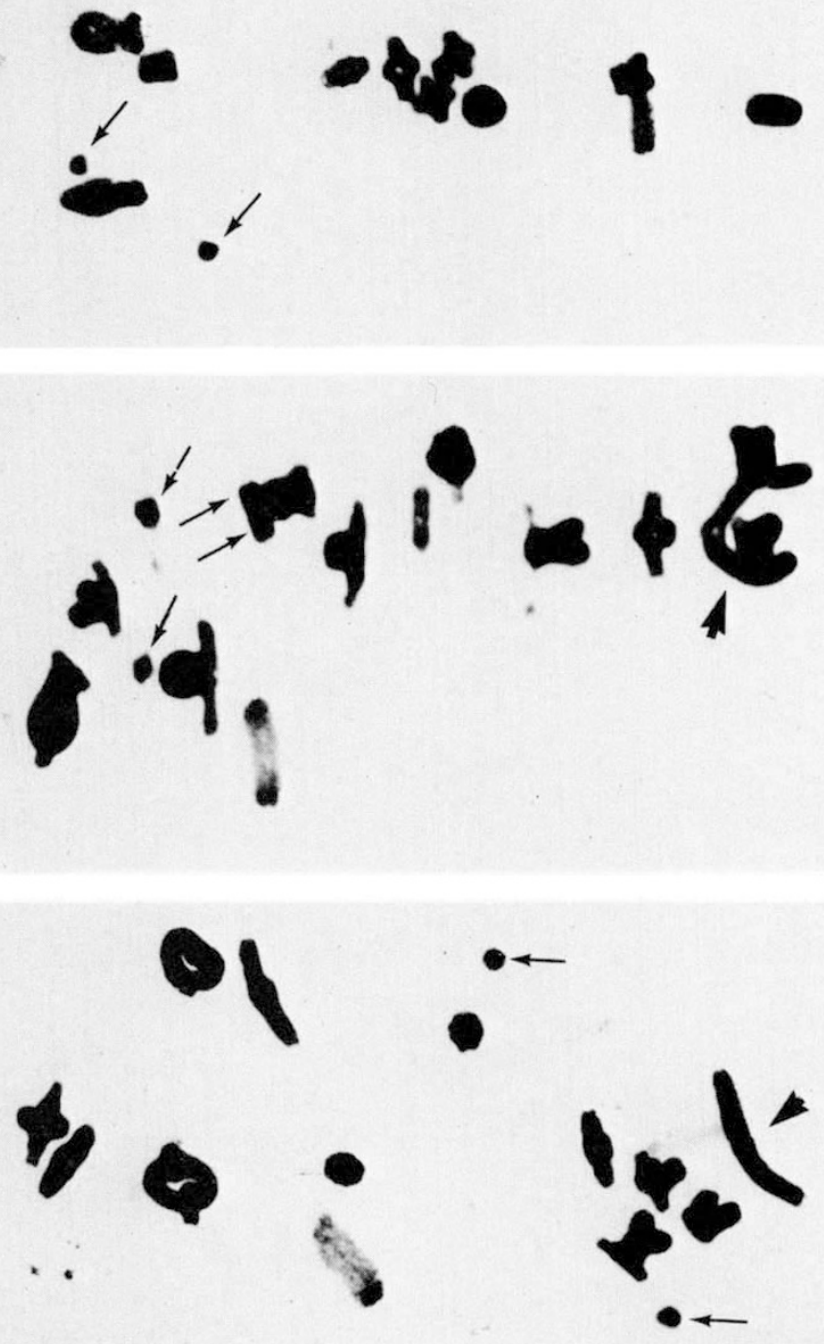

C

PLATE 1. Metaphase-I from a male of $A$. fusca which integrated the $B$ in its genome. (a) Two unpaired Bs. (b) Four $B s$ (arrows) plus a $\mathrm{L}_{2}$ trivalent (arrow head). (c) Two unpaired $B s$ (arrows) plus an extra $L_{2}$ bivalent (arrow head). 
TABLE 1

Populations of Arcyptera fusca and number of individuals with and without $\mathrm{B}$ s collected during seven consecutive generations in Respumoso

\begin{tabular}{|c|c|c|c|}
\hline \multirow[b]{2}{*}{ References } & \multicolumn{3}{|c|}{ Sample } \\
\hline & $O B$ & Bs & Tota \\
\hline AF.IV 1976 & 21 & 20 & 41 \\
\hline AF.IV 1977 & 10 & 9 & 19 \\
\hline AF.IV 1978 & 11 & 11 & 22 \\
\hline AF.IV 1979 & 21 & 18 & 39 \\
\hline AF.IV 1980 & 23 & 13 & 36 \\
\hline AF.IV 1981 & 15 & 10 & 25 \\
\hline AF.IV 1982 & 27 & 18 & 45 \\
\hline Total & 128 & 99 & 227 \\
\hline
\end{tabular}

Given the frequency of $B s$ in Respumoso, it was expected that 50 per cent of these two inoculations would contain $B$ s in their genomes. In both cases only males were used, so the incorporation of $B$ s into the genepool would be through non $B$ females of Pto. de la Bonaigua.

The karyotype analysis of the individuals from the 1979 generation (G79) did not reveal the existence of individuals carrying $B \mathrm{~s}$, although it does not entirely exclude the possibility that some $B$ individuals were not detected since a sample of males were used. However in 1980 (G80) two out of forty males collected had $B$ s with similar characteristics to those found in males from Respumoso (plate 1). Interfollicular variation in the number of $B \mathrm{~s}$, due to premeiotic accumulation $(0 B \mathrm{~s}, 1 B, 2 B \mathrm{~s}, 3 B \mathrm{~s}$ and $4 B \mathrm{~s}$ ) was also observed. Samples collected during 1981 (32 individuals) and 1982 (25 individuals) did not contain $\boldsymbol{B}$ s and the frequency of $\boldsymbol{B}$-carrying individuals at the Pto. de la Bonaigua is now probably zero.

In one of the two individuals found with Bs at Pto. de la Bonaigua, the $\mathrm{L}_{2}$ chromosome was polysomic in all cells of four of the testis follicles. The extra $\mathrm{L}_{2}$ chromosome could pair and form a single bivalent, usually with a distal chiasma (plate lc) or form multiples with the standard pair also by means of a terminal chiasma (plate $2 b$ ). The fact that such an anomaly was only found in this individual out of over four hundred individuals studied from this and other populations led us to wonder if it might be related to genetic divergence between the populations studied. Such divergence is reasonable because of the restricted mobility in the females due to the small size of the termina and wings, the unfavorability of the intervening terrain, where high mountains break the continuity of the distribution, and finally the relatively short life of the species which might contribute to a restriction in the genetic interchange between populations.

The major problem in discussing the present experiment is the partially negative results obtained. Although some of the introduced males were effective in impregnating females, it is difficult to know for certain the causes which produced the elimination in the next generation. However, the evidence that the $B$ could be introduced, at least in one generation, indicates that postzygotic mechanisms of selection rather than prezygotic ones might 
be effective in the elimination of the $B$. The stabilisation of a $B$-chromosome in a population could depend, as in the grasshopper Myrmeleotettix maculatus, on various factors such as meiotic drive in egg cells (Hewitt, 1973), selection against certain karyotypes carrying $B$ s and the selective advantage of others. The genome of the recipient population could be different and not interact with the introduced $B$ to produce a stable equilibrium as in the donor population. It is also possible that a relatively ineffective inoculation would permit drift alone to eliminate the $B$ s rather easily.

Acknowledgements. We gratefully acknowledge the advice and criticism of Dr Michael Shaw and Dr José L. Oliver. Carlos García de la Vega made this work possible in different ways.

\section{REFERENCES}

HEWITT, G. M. 1973. The integration of supernumerary chromosomes into the Orthopteran genome. Cold. Spring. Harbour, Symp. Quant. Biol, 38, 183-194.

JONES, R. N. AND REES, H. 1982. B-Chromosomes. Academic Press. London. 266.

LOPEZ-FERNANDEZ, C. AND GOSALVEZ, J. 1981. Differential staining of a heterochromatic zone in Arcyptera fusca (Orthoptera). Experientia, 37, 240.

LOPEZ-FERNANDEZ, C. AND GOSALVEZ, J. 1983. The chromosome system in three species of the genus Arcyptera (Orthoptera: Acrididae). II. Unstable B-chromosomes in Arcyptera fusca. Genetica, 62, 41-46.

LOPEZ-FERNANDEZ, C., GARCIA DE LA VEGA, C. AND GOSALVEZ, J. 1983. Spontaneous supernumerary isochromosomes in the grasshopper Acryptera fusca (Pall). Cytobios, 37, $107-111$.

LUCOV, z. AND NUR, U. 1973. Accumulation of $B$-chromosomes by preferential segregation in females of the grasshopper Melanoplus femur-rubrum. Chromosoma, 42, 278-306.

SHAW, M. 1983a. Rapid movement of a $B$-chromosome frequency cline in Myrmeleotettix maculatus (Orthoptera: Acrididae) Heredity, 50, 1-14.

SHAW, M. $1983 \mathrm{~b}$. Movement of a cline for supernumerary chromosomes. Kew Chromosome Conference II., 1982, 217-223. George Allen \& Unwin. 